\title{
Exhaled nitric oxide levels and blood eosinophil counts independently associate with wheeze and asthma events in National Health and Nutrition Examination Survey subjects
}

\author{
Andrei Malinovschi, MD, PhD, ${ }^{a}$ João A. Fonseca, MD, PhD, ${ }^{b}$ Tiago Jacinto, MSc, ${ }^{b}$ Kjell Alving, PhD, ${ }^{c *}$ and \\ Christer Janson, MD, PhD ${ }^{\mathbf{d} *}$ Uppsala, Sweden, and Porto, Portugal
}

Background: Fraction of exhaled nitric oxide (FENO) and blood eosinophil count (B-Eos) values, markers of local and systemic eosinophilic inflammation, respectively, are increased in asthmatic patients. Little is known about the relation of these markers to reported wheeze and asthma events in a random population sample. Objectives: We sought to determine the individual and independent values of B-Eos and Feno in relation to wheeze, asthma diagnosis, and asthma events in a cross-sectional study. Methods: Feno and B-Eos values were measured in 12,408 subjects aged 6 to 80 years from the National Health and Nutrition Examination Survey 2007-2008 and 2009-2010. Current wheeze and asthma diagnosis, as well as asthma attacks and asthma-related emergency department (ED) visits within the last 12 months, were assessed by means of questionnaires. Results: Intermediate or high FENO values and intermediate or high B-Eos values were independently associated with having asthma, wheeze, and asthma attacks. However, only intermediate and high B-Eos values were independently associated with asthma-related ED visits. High FENO ( $\geq 50$ ppb) and B-Eos $\left(\geq 500\right.$ cells $/ \mathrm{mm}^{3}$ ) values rendered an adjusted odds ratio of 4.5 of having wheeze, 5.1 of having asthma, 5.4 for asthma attacks, and 2.9 for asthma-related ED visits compared with normal FeNO ( $<25 \mathrm{ppb})$ and B-Eos $\left(<300\right.$ cells $\left./ \mathrm{mm}^{3}\right)$ values. Conclusions: Exhaled nitric oxide and B-Eos values offered independent information in relation to the prevalence of wheeze, asthma diagnosis, and asthma events in this random population sample. The clinical importance of these findings in asthmatic patients with regard to phenotyping and individualized treatment, considering both local and systemic eosinophilic inflammation, needs to be determined. (J Allergy Clin Immunol 2013;132:821-7.)

Key words: Asthma, wheeze, exhaled nitric oxide, blood eosinophils, National Health and Nutrition Examination Survey, inflammation

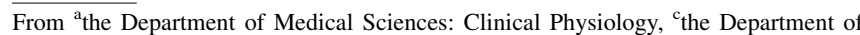
Women's and Children's Health, and dhe Department of Medical Sciences: Respiratory Medicine and Allergology, Uppsala University, and ${ }^{\mathrm{b}}$ CINTESIS, Faculdade de Medicina da Universidade do Porto \& Instituto e Hospital CUF Porto.

*These authors contributed equally to this work.

A.M. was supported by Uppsala University Hospital.

Disclosure of potential conflict of interest: J. A. Fonseca has received payment for lectures from Aerocrine AB, Merck, and Novartis. K. Alving is employed by and receives stock/stock options from Aerocrine $\mathrm{AB}$. The rest of the authors declare that they have no relevant conflicts of interest.

Received for publication October 3, 2012; revised June 3, 2013; accepted for publication June 5, 2013

Available online July 26, 2013

Corresponding author: Andrei Malinovschi, MD, PhD, Department of Medical Sciences: Clinical Physiology, Uppsala University, Akademiska Sjukhuset, 75185 Uppsala, Sweden. E-mail: Andrei.Malinovschi@medsci.uu.se.

0091-6749/\$36.00

(C) 2013 American Academy of Allergy, Asthma \& Immunology

http://dx.doi.org/10.1016/j.jaci.2013.06.007
}

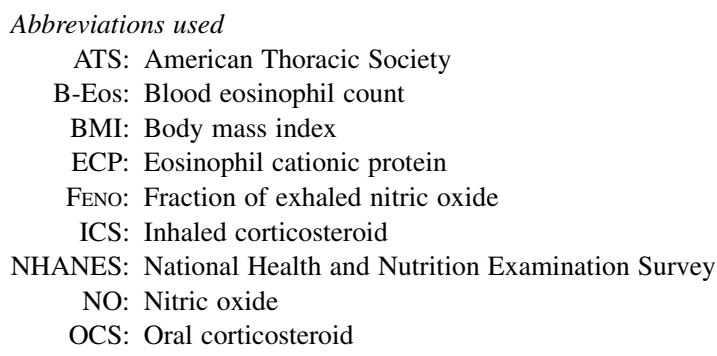

Discuss this article on the JACI Journal Club blog: www.jacionline.blogspot.com.

Asthma is characterized by chronic airway inflammation, variable airway obstruction, and airway hyperresponsiveness. ${ }^{1} \mathrm{In}$ flammation is a major component of asthma, and medication to control asthma is primarily anti-inflammatory. Nonetheless, it is only during the past decade that inflammometry (ie, measurement of inflammation to adjust anti-inflammatory therapy) has been introduced. $^{2}$ The fraction of exhaled nitric oxide (FENO) is a local marker of airways inflammation, primarily that triggered by IL4 and IL-13. ${ }^{3}$ Measurement of FENO is increasingly used in clinical practice, and clinical guidelines on the use of FENO have recently been published by the American Thoracic Society (ATS). ${ }^{4}$

Systemic eosinophilic inflammation, which is measured as blood eosinophil count (B-Eos) or serum eosinophil cationic protein (ECP) values, is also seen in asthmatic patients. Eosinophilia is primarily triggered by IL-5. ${ }^{7}$ Systemic eosinophilic inflammation is often regarded as a spillover from the inflamed airways. However, only moderate correlations have been reported between the 2 components, ${ }^{8}$ and this view is now being challenged by a model in which systemic inflammation plays an independent role in patients with asthma and other respiratory diseases. ${ }^{3,9,10}$ This new hypothesis is supported by data from studies showing that systemic inflammation is not always reduced by inhaled corticosteroids ${ }^{11,12}$ and that systemic therapies, such as leukotriene receptor antagonists, might be more effective in reducing systemic inflammatory markers. ${ }^{13,14}$ Furthermore, biological treatments directed at blocking IL-5 effectively decrease the signs of systemic inflammation, as measured by B-Eos values, but does not change FENO values, ${ }^{15}$ whereas anti-IL-13 treatment reduces FENO values without decreasing B-Eos values. ${ }^{16}$ Therefore it is reasonable to suggest that assessing both local and systemic eosinophilic inflammation in asthmatic patients would provide complementary information.

The aim of the present study was to assess the levels of local (FENO) and systemic (B-Eos) $\mathrm{T}_{\mathrm{H}} 2$ cytokine-driven inflammation in subjects from the US National Health and Nutrition 
TABLE I. Observed prevalence rates (percentages) of current asthma, wheeze, and asthma attack and asthma-related ED visits according to different FENO and B-Eos values (normal-intermediate-high)

\begin{tabular}{|c|c|c|c|c|c|c|c|c|c|}
\hline & No. & Current asthma & $P$ value* & Current wheeze & $P$ value* & Asthma attack & $P$ value* & $\begin{array}{c}\text { Asthma-related } \\
\text { ED visit in last year }\end{array}$ & $P$ value* \\
\hline Normal Feno value & 10,131 & $7.1 \%$ & $<.001$ & $11.7 \%$ & $<.001$ & $3.4 \%$ & $<.001$ & $1.1 \%$ & $<.001$ \\
\hline Intermediate FENO value & 1,688 & $10.7 \%$ & & $14.2 \%$ & & $5.6 \%$ & & $1.4 \%$ & \\
\hline Normal B-Eos value & 9,036 & $6.8 \%$ & $<.001$ & $10.7 \%$ & $<.001$ & $3.0 \%$ & $<.001$ & $0.8 \%$ & $<.001$ \\
\hline Intermediate B-Eos value & 2,499 & $10.3 \%$ & & $16.9 \%$ & & $5.8 \%$ & & $1.7 \%$ & \\
\hline High B-Eos value & 873 & $18.1 \%$ & & $21.8 \%$ & & $10.8 \%$ & & $3.4 \%$ & \\
\hline
\end{tabular}

*Pearson $\chi^{2}$ test statistics and associated probabilities ( $P$ values) were calculated to evaluate differences among observed percentages across normal-intermediate-high categories of FENO and B-Eos values.

Examination Survey (NHANES) ${ }^{17}$ and to investigate the relation between these 2 inflammatory components in respect to selfreported wheeze, physician-diagnosed asthma, asthma attacks, and emergency department (ED) visits for asthma.

\section{METHODS \\ Ethics statement}

The Ethics Review Board of the National Center for Health Statistics Research approved all protocols. All participants provided written informed consent.

\section{Study population}

The study included 12,408 participants aged 6 to 80 years from the NHANES 2007-2008 and NHANES 2009-2010 on whom exhaled nitric oxide (NO) measurements and blood differential counts had been performed Through the use of a complex probability cluster design, NHANES collects nationally representative cross-sectional data on the health status of the civilian, noninstitutionalized US population (http://www.cdc.gov/nchs/ nhanes.htm). Standardized health measurements were performed in mobile examination centers.

\section{Exhaled NO}

FENO values were measured with an electrochemical analyzer (NIOX $\mathrm{MINO}$; Aerocrine AB, Solna, Sweden) at an expiratory flow rate of $50 \mathrm{~mL} / \mathrm{s}$ in accordance with ATS/European Respiratory Society recommendations. ${ }^{18}$ The mean of 2 reproducible FENO measurements (within $2 \mathrm{ppb}$ if levels were $<30 \mathrm{ppb}$ or within $10 \%$ if levels were $>30 \mathrm{ppb}$ ) was taken as the final result.

Two measurements of less than the detection limit of the device $(<5 \mathrm{ppb})$ were considered reproducible and given an arbitrary value of $3.5 \mathrm{ppb}(1180$ subjects). Two measurements of greater than the detection limit of the device $(>300 \mathrm{ppb})$ were also considered reproducible and given an arbitrary value of $301 \mathrm{ppb}$ (1 subject).

In accordance with the ATS clinical guidelines on FENo measurement, ${ }^{4}$ we have designated levels of less than $20 \mathrm{ppb}$ (if $<12$ years of age) or less than 25 ppb as normal Feno levels (if $\geq 12$ years of age), levels of 20 or greater but less than $35 \mathrm{ppb}$ (if $<12$ years of age) and levels of 25 or greater but less than $50 \mathrm{ppb}$ (if $\geq 12$ years of age) as intermediate Feno levels, and levels of $35 \mathrm{ppb}$ or greater (if $<12$ years of age) or $50 \mathrm{ppb}$ or greater (if $\geq 12$ years of age) as high FENo levels.

\section{B-Eos}

Blood differential counts were performed in NHANES 2007-2008 and 2009-2010 by using the Beckman Coulter HMX (Beckman Coulter, Fullerton, Calif), a quantitative and automated hematologic analyzer and leukocyte differential cell counter for in vitro diagnostic use in clinical laboratories. A detailed description of the laboratory methods can be found on the NHANES Web site (http://www.cdc.gov/nchs/nhanes.htm).
The following cutoffs for B-Eos values were used: less than 300 cells $/ \mathrm{mm}^{3}$ to define normal B-Eos values, ${ }^{19} 300$ cells $/ \mathrm{mm}^{3}$ or greater but less than 500 cells $/ \mathrm{mm}^{3}$ for intermediate values, and 500 cells $/ \mathrm{mm}^{3}$ or greater for high B-Eos values, because this is the limit for defining eosinophilia. ${ }^{20}$

\section{Current asthma and current wheeze}

Current wheeze was defined as self-reported wheezing or whistling in the chest during the last 12 months. Current asthma was defined as self-reported physician-diagnosed asthma at any time of life together with a positive answer to the following question: "Do you still have asthma?" Asthma attacks and asthma-related ED visits during the last 12 months were self-reported.

\section{Hay fever}

Self-reported hay fever episodes in the past 12 months were considered to imply atopy. ${ }^{17}$

\section{Current use of inhaled or oral corticosteroids}

Self-reported use of inhaled corticosteroids (ICSs) or oral corticosteroids (OCSs) during the last 2 days before exhaled NO measurements was regarded as current use of corticosteroids.

\section{Smoking history}

Questions regarding cigarette use were only administered to participants aged 20 years and older. We assumed that younger participants were never smokers. ${ }^{17}$ A person was considered to be a never smoker if he or she had smoked less than 100 cigarettes in his or her entire life. Subjects reporting ever smoking at least 100 cigarettes during their lifetimes were classified, based on self-reported current smoking, as exsmokers or current smokers.

\section{Body mass index}

Height and weight were measured with digital stadiometers and scales, as described on the NHANES Web site (http://www.cdc.gov/nchs/nhanes.htm). Body mass index (BMI) was calculated as weight (kilograms) divided by height squared (square meters).

\section{Statistical analyses}

All the statistical analyses were conducted with STATA 12.1 software (StataCorp, College Station, Tex). Pearson correlation was used to study the relation between log-transformed FENO and B-Eos values. Pearson $\chi^{2}$ test statistics and associated probabilities ( $P$ values) were calculated to evaluate differences among observed percentages across normal-intermediate-high categories of Feno and B-Eos values (Table I and see Tables E1 and E2 in this article's Online Repository at www.jacionline.org). Logistic regression models, having current asthma, wheeze, asthma attacks, or asthma-related ED visits as outcomes and FENO and B-Eos values as determinants, were used to calculate odds ratios and CIs for prevalence rates of current asthma, 
TABLE II. Factors associated with current asthma, wheeze, asthma attack, and asthma-related ED visit (adjusted odds ratio [95\% $\mathrm{Cl}])$

\begin{tabular}{lcccc}
\hline & Current asthma & Wheeze & Asthma attack & Asthma-related ED visit \\
\hline Female sex & $1.47(1.27-1.72)$ & $1.09(0.97-1.23)$ & $1.52(1.24-1.88)$ & $1.49(1.04-2.14)$ \\
Age per 10 y & $0.79(0.75-0.82)$ & $0.92(0.89-0.95)$ & $0.77(0.72-0.82)$ & $0.77(0.68-0.86)$ \\
FENO value & & & & \\
$\quad$ Intermediate* & $1.50(1.22-1.85)$ & $1.30(1.09-1.54)$ & $1.44(1.08-1.91)$ & $1.16(0.70-1.91)$ \\
$\quad$ High* & $2.37(1.81-3.12)$ & $2.23(1.77-2.83)$ & $2.32(1.64-3.27)$ & $1.12(0.60-2.10)$ \\
B-Eos value & $1.26(1.05-1.51)$ & $1.39(1.21-1.59)$ & $1.60(1.26-2.03)$ & $1.71(1.13-2.59)$ \\
$\quad$ Intermediate* & $1.86(1.45-2.38)$ & $1.66(1.35-2.04)$ & $2.19(1.59-3.01)$ & $2.58(1.53-4.35)$ \\
$\quad$ High* & $1.20(1.14-1.26)$ & $1.19(1.14-1.24)$ & $1.20(1.13-1.28)$ & $1.22(1.11-1.35)$ \\
BMI per 5 units & $2.46(2.06-2.93)$ & $2.39(2.08-2.75)$ & $2.45(1.94-3.08)$ & $1.12(0.73-1.73)$ \\
Hay fever & & & $1.16(0.83-1.61)$ & $1.33(0.76-2.34)$ \\
Smoking history & $1.15(0.91-1.46)$ & $1.58(1.32-1.89)$ & $1.33(1.00-1.76)$ & $1.41(0.88-2.27)$ \\
$\quad$ Exsmoker $\dagger$ & $1.23(1.00-1.52)$ & $3.66(3.17-4.23)$ & \\
$\quad$ Current $\dagger$ & & &
\end{tabular}

Multiple logistic regression analyses were performed, and adjusted ORs (95\% CIs) are presented. A relation is considered significant if the CI does not include 1.

*Compared with normal Feno or B-Eos values, respectively.

†Compared with never smokers.

TABLE III. Adjusted odds ratios (95\% Cls) of current asthma, wheeze, asthma attack, and asthma-related ED visit with increased FENO and B-Eos values after adjusting for age, sex, BMI, hay fever, smoking history, and recent use of ICSs or OCSs

\begin{tabular}{llrcccc}
\hline Feno value & B-Eos value & No. & Current asthma & Wheeze & Asthma attack & Asthma-related ED visit \\
\hline Normal & Normal & 7,827 & 1 & 1 & 1 & 1 \\
Normal & Intermediate & 1,806 & $1.31(1.05-1.62)$ & $1.40(1.20-1.65)$ & $1.56(1.17-2.08)$ & $1.51(0.93-2.45)$ \\
Normal & High & 498 & $1.82(1.30-2.55)$ & $1.44(1.10-1.89)$ & $2.07(1.34-3.20)$ & $2.15(1.08-4.28)^{*}$ \\
Intermediate & Normal & 1,042 & $1.55(1.19-2.04)$ & $1.29(1.03-1.62)$ & $1.41(0.95-2.09)$ & $0.75(0.32-1.78)$ \\
Intermediate & Intermediate & 478 & $2.00(1.43-2.81)$ & $1.74(1.32-2.30)$ & $2.34(1.51-3.63)$ & $2.33(1.13-4.80)^{*}$ \\
Intermediate & High & 168 & $2.27(1.36-3.77)$ & $2.26(1.46-3.50)$ & $3.01(1.64-5.52)$ & $3.43(1.41-8.34)$ \\
High & Normal & 167 & $2.51(1.50-4.18)$ & $1.81(1.15-2.84)$ & $1.82(0.84-3.98)$ & $0.73(0.10-5.45)$ \\
High & Intermediate & 215 & $2.41(1.56-3.71)$ & $2.86(2.00-4.11)$ & $3.72(2.28-6.08)$ & $1.86(0.75-4.61)$ \\
High & High & 207 & $5.14(3.54-7.45)$ & $4.49(3.18-6.33)$ & $5.36(3.42-8.42)$ & $2.91(1.36-6.20)$ \\
\hline
\end{tabular}

Multiple logistic regression analyses were performed, and adjusted ORs (95\% CIs) are presented. All strata are compared with the stratum with normal FENO and B-Eos values. A relation is considered significant if the $\mathrm{CI}$ does not include 1.

*Significance not consistent after adjusting for multiple comparisons (Holm method).

TABLE IV. Population characteristics $(n=12,408)$

\begin{tabular}{lc}
\hline Outcomes & $1,032(8.3 \%)$ \\
Current asthma & $1,577(12.7 \%)$ \\
Wheeze during last year & $506(4.1 \%)$ \\
Asthma attacks in last year & $148(1.2 \%)$ \\
Asthma-related ED visits in last year & \\
Dependent variables & $13(3.5-301)$ \\
FENo value $(\mathrm{ppb})$ & $0.2(0-8.4)$ \\
B-Eos value $\left(\times 10^{9} / \mathrm{L}\right)$ & \\
Independent variables & $36(6-80)$ \\
Age $(\mathrm{y})$ & $6,080(49 \%)$ \\
Female sex & $26.3(12.5-84.9)$ \\
BMI $\left(\mathrm{kg} / \mathrm{m}^{2}\right)$ & $2,087(17.2 \%)^{*}$ \\
Past smoking & $2,087(17.2 \%)^{*}$ \\
Current smoking & $1,688(13.7 \%)$ \\
Hay fever & $491(4.0 \%)$ \\
Use of ICSs or OCSs in last $2 \mathrm{~d}$ &
\end{tabular}

Values are presented as medians (ranges) or numbers (percentages).

*Calculated for subjects older than 20 years for whom data were available on cigarette use.

wheeze, asthma attacks, or asthma-related ED visits (Table II and see Tables E3 and E4 in this article's Online Repository at www.jacionline.org). FENO and B-Eos values were included in the regression models a priori. A similar model with the same outcomes (current asthma, wheeze, asthma attacks, or

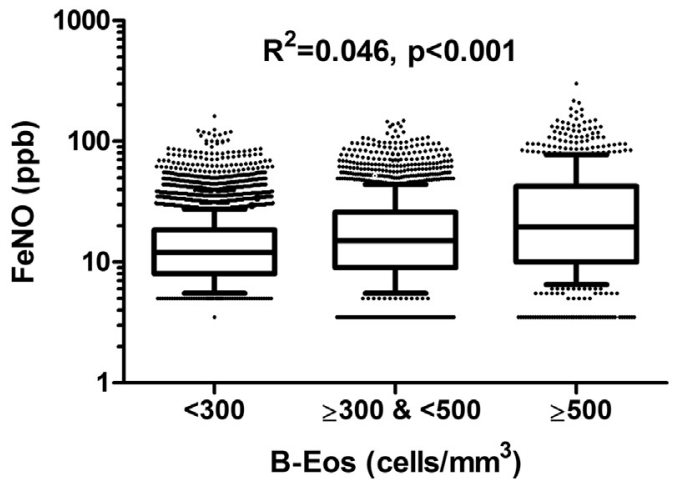

FIG 1. Feno values in subjects with normal, intermediate, or high B-Eos values. Line, Median; box, 25th-75th percentile; whiskers, 10th and 90th percentiles; outliers, below 10th and above 90th percentiles.

asthma-related ED visits) and a combination of FENO and B-Eos value categories as a determinant was used in Table III and Table E5 in this article's Online Repository at www.jacionline.org. These multiple logistic regression models included adjustments for age, sex, BMI, hay fever, current smoking, and recent use of inhaled or oral corticosteroids. Holm adjustments for multiple comparisons were also reported in the models described in Tables III and E5. Because atopy is related to Feno values, ${ }^{21}$ a multiple logistic regression 

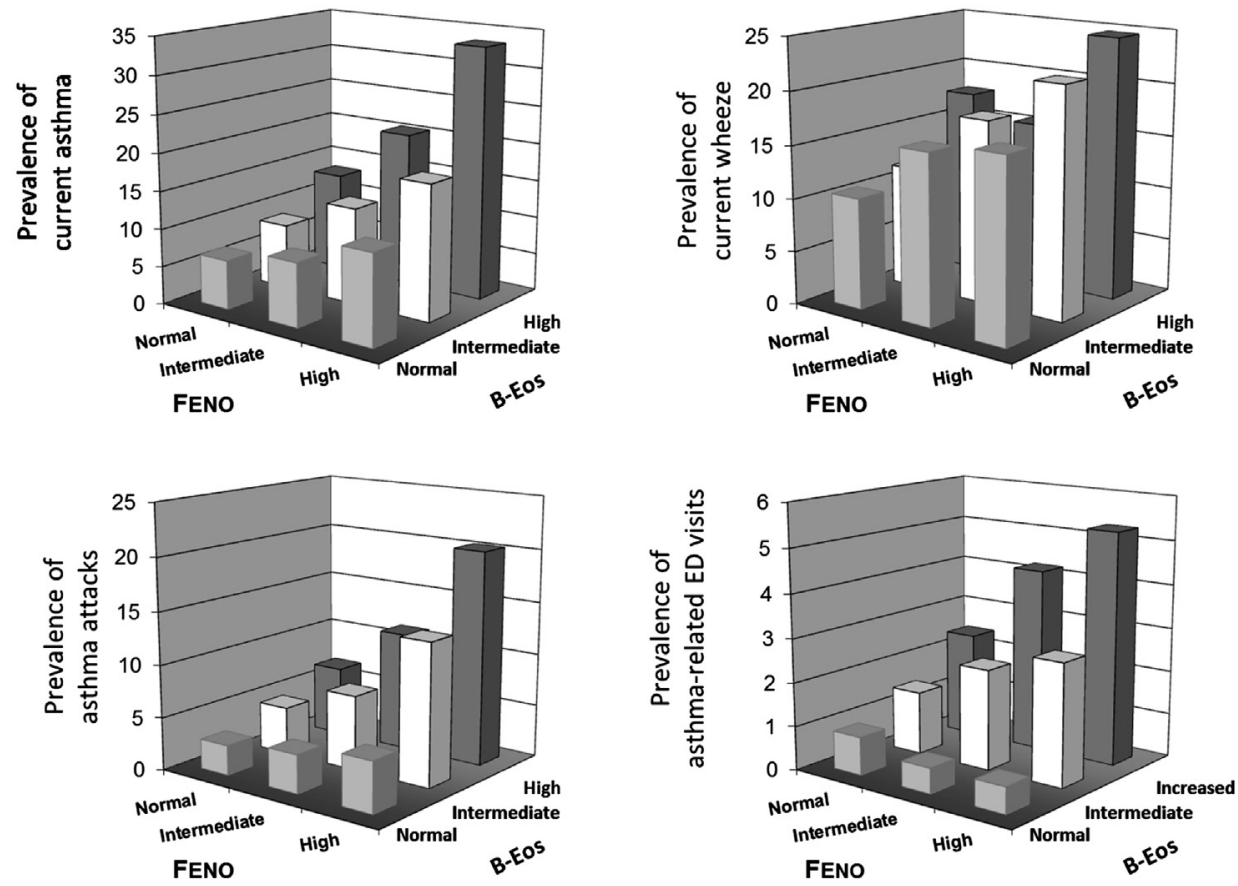

Number of subjects per group ( $\mathrm{n}$ ), according to levels of FENO and B-Eos.

\begin{tabular}{|c|c|c|c|}
\hline 7,827 & 1,042 & 167 & Normal B-Eos \\
\hline 1,806 & 478 & 215 & Intermediate B-Eos \\
\hline 498 & 168 & 207 & High B-Eos \\
\hline Normal FENO & Intermediate FENO & High FENO & \\
\hline
\end{tabular}

FIG 2. Prevalence of current asthma (left upper panel), current wheeze (right upper panel), asthma attacks (left lower panel), and ED visits (right lower panel) according to normal, intermediate, or high FENO and blood eosinophil values. The number of subjects in each category is presented in the table above.

model not adjusted for hay fever (used as proxy for atopy in this study) was also reported (see Table E5). A $P$ value of less than .05 was considered statistically significant.

\section{RESULTS}

A total of 12,408 subjects have performed FENO measurements and blood differential counts and were included in the study. The characteristics of these subjects are presented in Table IV.

There was a weak but statistically significant correlation between the Feno and B-Eos values (Pearson $r=0.22$, $P<.001)$. The explanatory value $\left(R^{2}\right)$ was $4.0 \%$. Feno values, according to different (normal-intermediate-high) B-Eos value categories, are depicted in Fig 1, and the explanatory value $\left(R^{2}\right)$ of this model was $4.6 \%$. The explanatory value was slightly higher when looking at the subgroups of subjects with current asthma (n $\left.=994, R^{2}=0.13, P<.001\right)$ or wheeze (n $=1519$, $\left.R^{2}=0.09, P<.001\right)$.

The prevalence of current asthma and wheeze increased progressively with increased FENO values (Table I). A similar increase in current asthma and wheeze was observed with increased B-Eos values (all $P<.001$, Table I). Also, the prevalence of asthma attacks and asthma-related ED visits increased with higher FENO and B-Eos values, respectively (Table I). These results were consistent when separately analyzing children (see Table E1) and adults (see Table E2).

When looking at the 2 markers in combination, the prevalence of current asthma increased with increased FENO and B-Eos values from $6.2 \%$ in the group with normal FENO and B-Eos values to $33.3 \%$ in the group with high FeNO and B-Eos values (Fig 2). Having intermediate or high FENO values and intermediate or high B-Eos values was independently associated with having asthma, wheeze, and asthma attacks (Table II). However, only intermediate and high B-Eos values were independently associated with asthma-related ED visits. Additional risk factors were high BMI, hay fever, and current smoking (Table II). Similar relations were found in children (see Table E3) and adults (see Table E4). No significant interactions with age (children vs adults) were found when analyzing the relation between having intermediate and high FENO values or intermediate and high B-Eos values and wheezing, asthma, and asthma events.

An increased probability of having current asthma and wheeze was found with increasing FENO values by different strata of B-Eos values (Fig 3), with the highest probability in the group with high B-Eos values. A significantly increased probability of asthma attacks was found with increased FENO values only in subjects with intermediate or high B-Eos values, whereas a significantly increased probability of asthma-related ED visits was found only in subjects with high B-Eos values.

The risk (odds ratio) of having wheeze or current asthma increased approximately 5-fold in subjects with both high FENO and high B-Eos values compared with subjects with normal FENO and normal B-Eos values after adjustment for other risk factors (Table III and see Table E5 without adjustment for hay fever). The corresponding odds ratios for asthma attacks and 

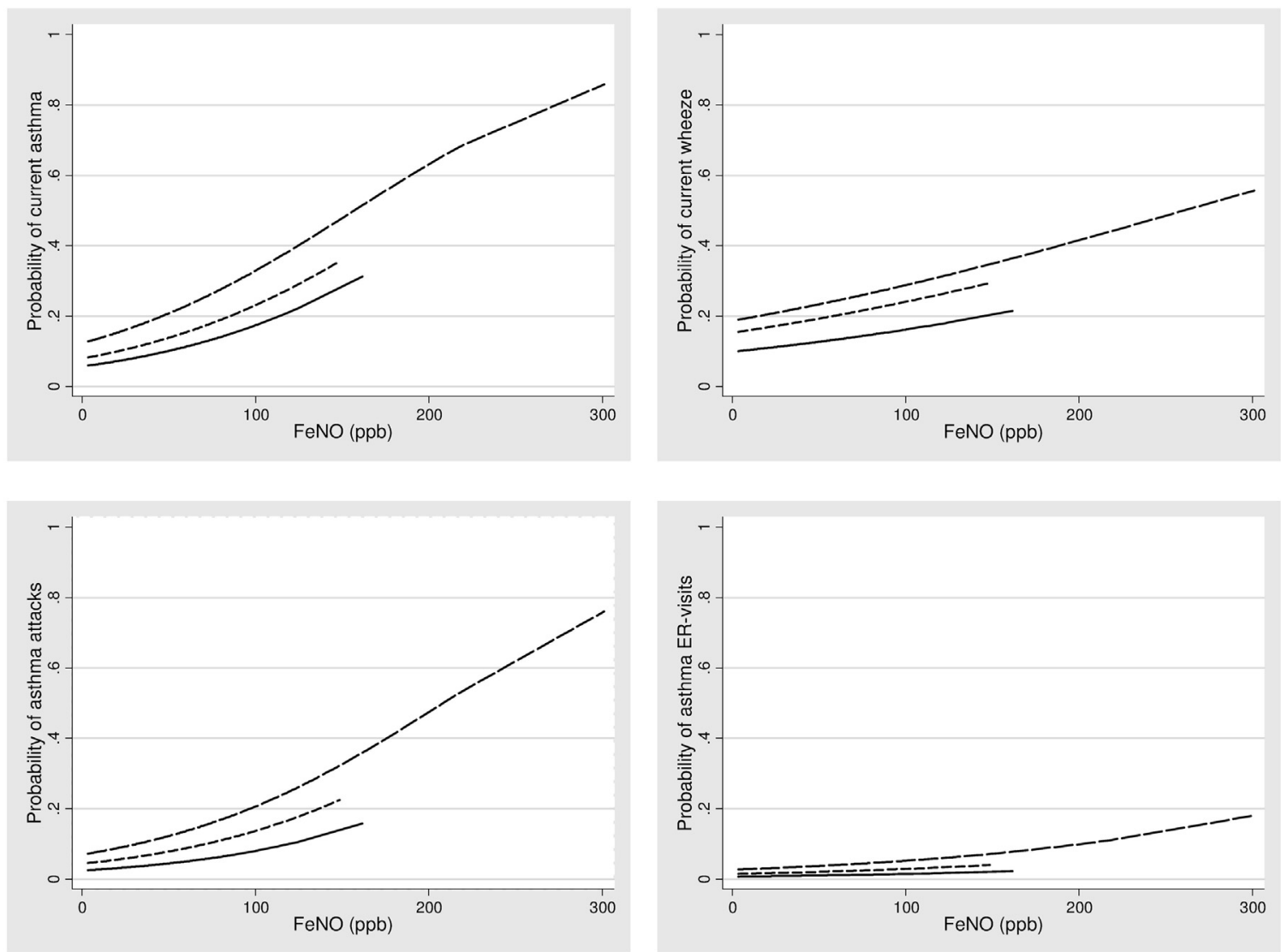

FIG 3. Probability of current asthma (left upper panel), current wheeze (right upper panel), asthma attacks (left lower panel), and ED visits (right lower panel) with increased FENo values plotted for each stratum of B-Eos values: normal (continuous line), intermediate (dashed line), and high (long-dashed line).

asthma-related ED visits were approximately 5 and 3, respectively. The risk of having an asthma-related ED visit was not significantly increased in subjects with high FENO values if they had normal or intermediate B-Eos values, whereas high B-Eos values were related to ED visits regardless of FENO values (Table III). A consistent effect of having both high FENO and high B-Eos values on all the asthma variables was seen after exclusion of subjects taking ICSs or OCSs and after the additional exclusion of subjects reporting hay fever. Furthermore, a consistent effect on wheeze was seen after excluding current asthma (Fig 4).

\section{DISCUSSION}

The main finding from this study in a large population was that FENO and B-Eos values were each independently associated with current asthma, wheeze, and asthma attacks, as well as being associated in an additive manner. Our data support the view that these 2 markers cannot be used interchangeably but should be used in combination. However, it must be recognized that this is a population-based study with the main purpose of identifying new information regarding these 2 inflammatory components and not a study for determining the diagnostic accuracy of these methods. Such a study should be performed in a more relevant population, such as in subjects undergoing clinical investigations because of suspected asthma.

In the present study, asthma and wheeze were 3 times more prevalent among subjects with high exhaled NO values than among subjects with normal values, and the prevalence was increased to a similar level among those with high B-Eos values. Several studies have previously found such associations for each of these markers separately, ${ }^{22}$ but to our knowledge, no earlier study has evaluated the additive predictive value of systemic eosinophil markers and exhaled NO values.

One reason why few have tested the predictive value of the combination of FENO and B-Eos might be that these markers are generally considered to measure the same inflammatory component of asthma, usually defined as "eosinophilic airway inflammation." ${ }^{4}$ However, in the present study the correlation between FENO and B-Eos values was weak, with an explanatory value of only approximately $4 \%$, which is in line with the previously reported weak-to-moderate correlations between FENO and eosinophil values in blood or sputum ${ }^{8,23}$ or between blood and sputum eosinophil values. ${ }^{24}$ This, together with the clear-cut additive effect of these 2 markers on the risk for asthma, wheeze, and asthma attacks in our study, indicates that they represent 2 different inflammatory pathways with separate trigger mechanisms.

Interestingly, increased blood eosinophil values were more important in relation to asthma-related ED visits in this material, which is in line with the recent findings of severe asthma exacerbation reductions in subjects with severe eosinophilic asthma $^{25}$ receiving anti-IL-5 treatment, which primarily targets the systemic eosinophilic inflammation. A previous study performed in children reported a relation between serum ECP values and asthma attacks, ${ }^{26}$ whereas a Dutch registry study reported a 

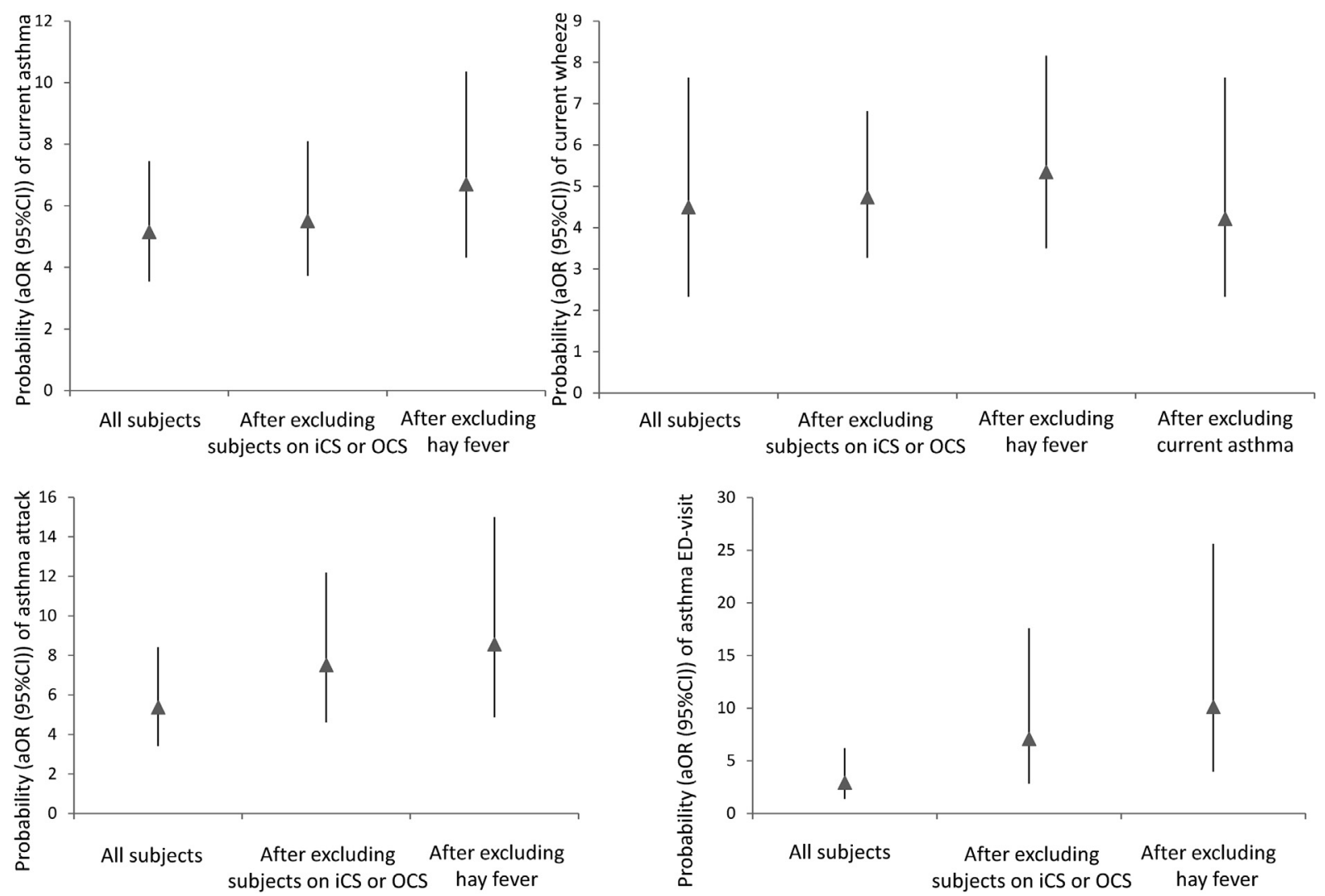

FIG 4. Probability (aOR [95\% CI]) of current asthma (left upper panel), current wheeze (right upper panel), asthma attacks (left lower panel), and ED visits (right lower panel) after exclusion of subjects taking ICSs or OCSs, those with hay fever, and those with current asthma (the latter only for the analyses in the right upper panel) in subjects with both high FENO and high B-Eos values (compared with subjects with normal FENo and normal B-Eos values).

relation between asthma attacks with eosinophilia and mortality. ${ }^{27}$ FENO signals local IL-4/IL-13-mediated mechanisms in the bronchial mucosa that are primarily triggered by aeroallergen exposure. $^{3}$ An increase in FENO values seems to precede moderate, but not severe, asthma exacerbations. ${ }^{28}$ We have recently reported that guiding anti-inflammatory treatment in asthma based on FENO measurement resulted in a reduction of moderate, but not severe, exacerbations. ${ }^{29}$ Severe exacerbations are primarily related to viral infections. ${ }^{30}$ Increased baseline sputum eosinophil values were related to more severe symptoms in respect to experimental rhinovirus infection, ${ }^{31}$ which might support our findings on the relation between increased B-Eos values and asthma-related ED visits.

The general view has been that eosinophilic asthma can be identified by at least 1 of the following: increased FENO, increased sputum eosinophil, or increased blood eosinophil values. ${ }^{18,25}$ Our results indicate that this view is valid only at the group level and not at the individual level. At the individual level, a combination of markers is preferred. Furthermore, from recent asthma studies in which the clinical effect of new biological drugs has been evaluated, particularly anti-IL- $5^{15}$ and anti-IL-13, ${ }^{16}$ it is evident that exhaled NO and blood eosinophil values signal different cytokine mechanisms. ${ }^{3}$ Blood eosinophilia is primarily driven by $\mathrm{IL}-5^{7}$ and might be triggered also, for example, by subchronic or persistent rhinovirus infections ${ }^{32}$ or in connection to chronic rhinosinusitis with or without polyposis ${ }^{33}$ in addition to aeroallergen exposure.
Taken together, both local and systemic $\mathrm{T}_{\mathrm{H}} 2$ cytokine-driven mechanisms, partly with different triggers, seem to be involved in "eosinophilic" asthma, suggesting a "double-hit" mechanism for the development of respiratory symptoms and asthma.

One advantage of FENO and B-Eos measurements is that these methods are relatively inexpensive and that the sampling causes less discomfort for the patients than other methods of assessing inflammation, such as induced sputum or bronchoscopy. During recent years, there has been a great interest in identifying different phenotypes of asthma to develop more targeted therapeutic interventions. ${ }^{33}$ Our data indicate that using FENO and B-Eos measurements in combination might be a cost-effective way of gathering phenotypic information in asthmatic patients ${ }^{34}$ and that the systemic eosinophilic component might be more related to severe asthma exacerbations, as discussed above.

Even though the combination of high FENO and B-Eos values was strongly related to current asthma, wheeze, and asthma attacks, it should be noted that a significant proportion of subjects in this group reported neither. However, it is possible that asymptomatic subjects with the combination of high FENO and high B-Eos values constitute a group with a very high risk of asthma because increased B-Eos values have been related to future development of asthma in a longitudinal study ${ }^{35}$ and increased serum ECP levels were related to the incidence of asthma in a population of patients with allergic rhinitis. ${ }^{36}$ Similarly, increased FENO values have been shown to indicate increased risk of asthma or wheeze. ${ }^{37,38}$ 
A possible limitation of the present study is that the biomarkers used are both markers of the eosinophilic type of inflammation, and therefore other inflammatory patterns, such as neutrophilic inflammation, would not be identified. However, in the general asthmatic population the phenotype characterized by eosinophilic activation predominates. ${ }^{33}$ Furthermore, the main result regarding the independent and additive value of the 2 biomarkers in detecting asthma or wheeze would probably not have been affected by the presence of noneosinophilic asthma. No data on IgE sensitization are available for the subjects included in the present study. However, the additive value of FENO and B-Eos measurements for predicting asthma or wheeze was confirmed, even after we excluded subjects with reported hay fever as a proxy for $\operatorname{IgE}$ sensitization.

In conclusion, information from this large population study shows that it might be advantageous to assess both local inflammation in the airways (FENO) and systemic eosinophilic inflammation (eg, B-Eos) to identify subjects with wheeze and asthma and patients at risk of asthma exacerbations. Our findings support the view that both local and systemic $\mathrm{T}_{\mathrm{H}} 2$ cytokine-driven mechanisms are important for the development of respiratory symptoms and clinical asthma, but the clinical use of the combination of these markers warrants further study.

Clinical implications: Local and systemic $\mathrm{T}_{\mathrm{H}} \mathbf{2}$ cytokine-driven
mechanisms independently trigger the development of respira-
tory symptoms and clinical asthma. The clinical importance of
assessing both these components for individualizing treatment
decisions warrants further study.

\section{REFERENCES}

1. Global Initiative for Asthma (GINA). Global strategy for asthma management and prevention. Available at: http://www.ginasthma.org/local/uploads/files/ GINA_Report_March13.pdf. Accessed June 27, 2013.

2. Pijnenburg MW, Jobsis Q, de Jongste JC. ['Inflammometry' with nitric oxide in exhaled air: a new test for lung diseases]. Ned Tijdschr Geneeskd 2001;145:946-50.

3. Alving K, Malinovschi A. Basic aspects of exhaled nitric oxide. European Respiratory Society Monograph 2010;49:1-31.

4. Dweik RA, Boggs PB, Erzurum SC, Irvin CG, Leigh MW, Lundberg JO, et al. An Official ATS clinical practice guideline: interpretation of exhaled nitric oxide levels (FENO) for clinical applications. Am J Respir Crit Care Med 2011;184:602-15.

5. Burrows B, Hasan FM, Barbee RA, Halonen M, Lebowitz MD. Epidemiologic observations on eosinophilia and its relation to respiratory disorders. Am Rev Respir Dis 1980;122:709-19.

6. Bjornsson E, Janson C, Hakansson L, Enander I, Venge P, Boman G. Serum eosinophil cationic protein in relation to bronchial asthma in a young Swedish population. Allergy 1994;49:730-6.

7. Kips JC, O'Connor BJ, Langley SJ, Woodcock A, Kerstjens HA, Postma DS, et al Effect of SCH55700, a humanized anti-human interleukin-5 antibody, in severe persistent asthma: a pilot study. Am J Respir Crit Care Med 2003;167:1655-9.

8. Strunk RC, Szefler SJ, Phillips BR, Zeiger RS, Chinchilli VM, Larsen G, et al. Relationship of exhaled nitric oxide to clinical and inflammatory markers of persistent asthma in children. J Allergy Clin Immunol 2003;112:883-92.

9. Holt PG, Sly PD. Viral infections and atopy in asthma pathogenesis: new rationales for asthma prevention and treatment. Nat Med 2012;18:726-35.

10. Wouters EF, Reynaert NL, Dentener MA, Vernooy JH. Systemic and local inflammation in asthma and chronic obstructive pulmonary disease: is there a connection? Proc Am Thorac Soc 2009;6:638-47.

11. Kips JC, Pauwels RA. Serum eosinophil cationic protein in asthma: what does it mean? Clin Exp Allergy 1998;28:1-3

12. Currie GP, Syme-Grant NJ, McFarlane LC, Carey FA, Lipworth BJ. Effects of low dose fluticasone/salmeterol combination on surrogate inflammatory markers in moderate persistent asthma. Allergy 2003;58:602-7.

13. Stelmach I, Jerzynska J, Kuna P. A randomized, double-blind trial of the effect of treatment with montelukast on bronchial hyperresponsiveness and serum eosinophilic cationic protein (ECP), soluble interleukin 2 receptor (sIL-2R), IL-4, and soluble intercellular adhesion molecule 1 (sICAM-1) in children with asthma. J Allergy Clin Immunol 2002;109:257-63.

14. Spahn JD, Covar RA, Jain N, Gleason M, Shimamoto R, Szefler SJ, et al. Effect of montelukast on peripheral airflow obstruction in children with asthma. Ann Allergy Asthma Immunol 2006;96:541-9.

15. Haldar P, Brightling CE, Hargadon B, Gupta S, Monteiro W, Sousa A, et al. Mepolizumab and exacerbations of refractory eosinophilic asthma. N Engl J Med 2009;360:973-84.

16. Corren J, Lemanske RF, Hanania NA, Korenblat PE, Parsey MV, Arron JR, et al Lebrikizumab treatment in adults with asthma. N Engl J Med 2011;365:1088-98

17. See KC, Christiani DC. Normal values and thresholds for the clinical interpretation of exhaled nitric oxide levels in the U.S. general population: results from NHANES 2007-2010. Chest 2013;143:107-16.

18. ATS/ERS recommendations for standardized procedures for the online and offline measurement of exhaled lower respiratory nitric oxide and nasal nitric oxide, 2005. Am J Respir Crit Care Med 2005;171:912-30.

19. Simmons A, Leaverton P, Elbert G. Normal laboratory values for differential white cell counts established by manual and automated cytochemical methods (Hemalog D-TM). J Clin Pathol 1974;27:55-8.

20. Gotlib J. World Health Organization-defined eosinophilic disorders: 2011 update on diagnosis, risk stratification, and management. Am J Hematol 2011;86:677-88.

21. Malinovschi A, Janson C, Holmkvist T, Norback D, Merilainen P, Hogman M. IgE sensitisation in relation to flow-independent nitric oxide exchange parameters. Respir Res 2006;7:92.

22. Nordvall SL, Janson C, Kalm-Stephens P, Foucard T, Toren K, Alving K. Exhaled nitric oxide in a population-based study of asthma and allergy in schoolchildren. Allergy 2005;60:469-75.

23. Mattes J, Storm van's GK, Reining U, Alving K, Ihorst G, Henschen M, et al. NO in exhaled air is correlated with markers of eosinophilic airway inflammation in corticosteroid-dependent childhood asthma. Eur Respir J 1999;13:1391-5.

24. Pizzichini E, Pizzichini MM, Efthimiadis A, Dolovich J, Hargreave FE. Measuring airway inflammation in asthma: eosinophils and eosinophilic cationic protein in induced sputum compared with peripheral blood. J Allergy Clin Immunol 1997;99:539-44.

25. Pavord ID, Korn S, Howarth P, Bleecker ER, Buhl R, Keene ON, et al. Mepolizumab for severe eosinophilic asthma (DREAM): a multicentre, double-blind, placebo-controlled trial. Lancet 2012;380:651-9.

26. Ishigaki N, Masuhara C, Sakamaki K, Ishikawa Y, Ohta K, Koike R, et al. [Relation between serum eosinophil cationic protein (ECP) level and asthma attack in children]. Arerugi 2000;49:1093-103.

27. Hospers JJ, Schouten JP, Weiss ST, Rijcken B, Postma DS. Asthma attacks with eosinophilia predict mortality from chronic obstructive pulmonary disease in a general population sample. Am J Respir Crit Care Med 1999;160:1869-74.

28. van der Valk RJ, Baraldi E, Stern G, Frey U, de Jongste JC. Daily exhaled nitric oxide measurements and asthma exacerbations in children. Allergy 2012;67: 265-71.

29. Syk J, Malinovschi A, Johansson G, Unden AL, Alving K. Lower incidence of asthma exacerbations with FENO-guided anti-inflammatory treatment: a randomised controlled trial. Eur Respir J 2012;40:797s.

30. Grissell TV, Powell H, Shafren DR, Boyle MJ, Hensley MJ, Jones PD, et al. Interleukin-10 gene expression in acute virus-induced asthma. Am J Respir Crit Care Med 2005; 172:433-9.

31. DeMore JP, Weisshaar EH, Vrtis RF, Swenson CA, Evans MD, Morin A, et al. Similar colds in subjects with allergic asthma and nonatopic subjects after inoculation with rhinovirus-16. J Allergy Clin Immunol 2009;124:245-52, e1-3.

32. Wos M, Sanak M, Soja J, Olechnowicz H, Busse WW, Szczeklik A. The presence of rhinovirus in lower airways of patients with bronchial asthma. Am J Respir Crit Care Med 2008;177:1082-9.

33. Wenzel SE. Asthma phenotypes: the evolution from clinical to molecular approaches. Nat Med 2012;18:716-25

34. Haldar P, Pavord ID, Shaw DE, Berry MA, Thomas M, Brightling CE, et al. Cluster analysis and clinical asthma phenotypes. Am J Respir Crit Care Med 2008;178 218-24.

35. Burrows B, Lebowitz MD, Barbee RA, Cline MG. Findings before diagnoses of asthma among the elderly in a longitudinal study of a general population sample. J Allergy Clin Immunol 1991;88:870-7.

36. Nielsen LP, Peterson CG, Dahl R. Serum eosinophil granule proteins predict asthma risk in allergic rhinitis. Allergy 2009;64:733-7.

37. Bastain TM, Islam T, Berhane KT, McConnell RS, Rappaport EB, Salam MT, et al. Exhaled nitric oxide, susceptibility and new-onset asthma in the Children's Health Study. Eur Respir J 2011;37:523-31.

38. Olin AC, Rosengren A, Thelle DS, Lissner L, Toren K. Increased fraction of exhaled nitric oxide predicts new-onset wheeze in a general population. Am J Respir Crit Care Med 2010;181:324-7. 
TABLE E1. Observed prevalence rates of current asthma, wheeze, and asthma events according to different FENO and B-Eos values (normal-intermediate-high [percentages]) among NHANES children ( $\mathrm{n}=3172$ )

\begin{tabular}{lrccccccc}
\hline & No. & Current asthma & $\boldsymbol{P}$ value* & Current wheeze & $\boldsymbol{P}$ value* & Asthma attack & $\begin{array}{c}\text { P value* } \\
\text { ED visit in last year }\end{array}$ & $\begin{array}{c}\boldsymbol{P} \text { value* } \\
\text { ED }\end{array}$ \\
\hline Normal FENO value & 2,535 & $9.1 \%$ & $<.001$ & $8.3 \%$ & $<.001$ & $4.2 \%$ & $<.001$ & $1.4 \%$ \\
Intermediate FENO value & 378 & $10.7 \%$ & & $14.8 \%$ & & $7.7 \%$ & .03 & $2.2 \%$ \\
High FENO value & 70 & $27.0 \%$ & & $24.7 \%$ & & $17.4 \%$ & $3.5 \%$ & $1.0 \%$ \\
Normal B-Eos value & 9,036 & $8.5 \%$ & $<.001$ & $7.7 \%$ & $<.001$ & $3.4 \%$ & $<.001$ & $<.001$ \\
Intermediate B-Eos value & 2,499 & $14.7 \%$ & & $14.0 \%$ & & $8.1 \%$ & $2.7 \%$ \\
High B-Eos value & 873 & $23.0 \%$ & & $20.7 \%$ & & $15.1 \%$ & $3.7 \%$ \\
\hline
\end{tabular}

* $P$ values for Pearson $\chi^{2}$ test. 
TABLE E2. Observed prevalence rates of current asthma, wheeze, and asthma events according to different FENO and B-Eos values (normal-intermediate-high [percentage]) among NHANES adults $(\mathrm{n}=9223$ )

\begin{tabular}{|c|c|c|c|c|c|c|c|c|c|}
\hline & No. & Current asthma & $P$ value* & Current wheeze & $P$ value* & Asthma attack & $P$ value* & $\begin{array}{l}\text { Asthma-related } \\
\text { ED visit in last year }\end{array}$ & $P$ value* \\
\hline Normal FENO value & 2,535 & $6.3 \%$ & $<.001$ & $12.9 \%$ & $<.001$ & $3.0 \%$ & $<.001$ & $0.9 \%$ & .006 \\
\hline Intermediate FENO value & 378 & $9.6 \%$ & & $14.1 \%$ & & $4.7 \%$ & & $1.2 \%$ & \\
\hline High FENO value & 70 & $19.7 \%$ & & $25.8 \%$ & & $10.3 \%$ & & $2.7 \%$ & \\
\hline Normal B-Eos value & 9,036 & $6.3 \%$ & $<.001$ & $11.6 \%$ & $<.001$ & $2.8 \%$ & $<.001$ & $0.8 \%$ & $<.001$ \\
\hline Intermediate B-Eos value & 2,499 & $8.8 \%$ & & $17.9 \%$ & & $5.0 \%$ & & $1.4 \%$ & \\
\hline High B-Eos value & 873 & $14.8 \%$ & & $22.5 \%$ & & $7.9 \%$ & & $3.3 \%$ & \\
\hline
\end{tabular}

${ }^{*} P$ values for Pearson $\chi^{2}$ test. 
TABLE E3. Factors associated with current asthma, wheeze, and asthma events (adjusted odds ratio [95\% CI]) in NHANES children $(n=3149)$

\begin{tabular}{|c|c|c|c|c|}
\hline & Current asthma & Wheeze & Asthma attack & Asthma-related ED visit \\
\hline Female sex & $0.89(0.69-1.15)$ & $0.78(0.61-1.01)$ & $0.78(0.55-1.10)$ & $0.78(0.43-1.42)$ \\
\hline Age per $10 \mathrm{y}$ & $0.73(0.47-1.13)$ & $0.78(0.50-1.20)$ & $0.44(0.25-0.80)$ & $0.24(0.08-0.66)$ \\
\hline \multicolumn{5}{|l|}{ FEno value } \\
\hline Intermediate* & $1.41(0.98-2.05)$ & $1.54(1.08-2.21)$ & $1.56(0.95-2.55)$ & $1.15(0.47-2.82)$ \\
\hline High* & $2.34(1.57-3.49)$ & $2.29(1.54-3.41)$ & $2.61(1.60-4.27)$ & $1.25(0.51-3.05)$ \\
\hline \multicolumn{5}{|l|}{ B-Eos value } \\
\hline Intermediate* & $1.38(1.00-1.89)$ & $1.37(1.01-1.88)$ & $1.65(1.08-2.52)$ & $2.00(0.98-4.06)$ \\
\hline High* & $1.81(1.23-2.67)$ & $1.61(1.10-2.38)$ & $2.36(1.45-3.83)$ & $2.07(0.89-4.85)$ \\
\hline BMI per 5 units & $1.36(1.22-1.53)$ & $1.25(1.12-1.41)$ & $1.38(1.18-1.60)$ & $1.48(1.15-1.90)$ \\
\hline Hay fever & $1.86(1.33-2.93)$ & $2.95(2.18-4.00)$ & $1.76(1.15-2.69)$ & $0.78(0.34-1.81)$ \\
\hline
\end{tabular}

Multiple logistic regression analyses were performed, and adjusted ORs (95\% CIs) are presented. A relation is significant if the CI does not include 1.

*Compared with normal FENO or B-Eos values, respectively. 
TABLE E4. Factors associated with current asthma, wheeze and asthma events (adjusted odds ratio [95\% CI]) in NHANES adults $(\mathrm{n}=8838)$

\begin{tabular}{|c|c|c|c|c|}
\hline & Current asthma & Wheeze & Asthma attack & Asthma-related ED visit \\
\hline Female sex & $2.07(1.70-2.52)$ & $1.22(1.06-1.40)$ & $2.34(1.78-3.08)$ & $2.24(1.37-3.67)$ \\
\hline Age per $10 \mathrm{y}$ & $0.90(0.84-0.95)$ & $0.97(0.93-1.02)$ & $0.85(0.78-0.93)$ & $0.86(0.74-1.00)$ \\
\hline \multicolumn{5}{|l|}{ FEno value } \\
\hline Intermediate* & $1.61(1.25-2.08)$ & $1.24(1.02-1.51)$ & $1.47(1.03-2.09)$ & $1.26(0.69-2.33)$ \\
\hline High* & $2.42(1.63-3.59)$ & $2.19(1.61-2.97)$ & $2.15(1.29-3.57)$ & $1.20(0.49-2.92)$ \\
\hline \multicolumn{5}{|l|}{ B-Eos value } \\
\hline Intermediate* & $1.15(0.91-1.44)$ & $1.35(1.16-1.58)$ & $1.51(1.12-2.04)$ & $1.49(0.89-2.52)$ \\
\hline High* & $1.68(1.19-2.38)$ & $1.53(1.19-1.98)$ & $1.65(1.04-2.63)$ & $2.58(1.30-5.15)$ \\
\hline BMI per 5 units & $1.23(1.16-1.30)$ & $1.21(1.16-1.27)$ & $1.22(1.13-1.32)$ & $1.25(1.11-1.40)$ \\
\hline Hay fever & $2.70(2.19-3.32)$ & $2.24(1.91-2.62)$ & $2.72(2.06-3.59)$ & $1.24(0.73-2.08)$ \\
\hline \multicolumn{5}{|l|}{ Smoking history } \\
\hline Exsmoker $\dagger$ & $1.31(1.03-1.67)$ & $1.68(1.41-2.02)$ & $1.30(0.93-1.82)$ & $1.46(0.81-2.62)$ \\
\hline Current $\dagger$ & $1.63(1.29-2.05)$ & $4.12(3.51-4.84)$ & $1.63(1.20-2.22)$ & $1.76(1.03-2.99)$ \\
\hline
\end{tabular}

Multiple logistic regression analyses were performed, and adjusted ORs (95\% CIs) are presented. A relation is significant if the CI does not include 1.

*Compared with normal FENo or B-Eos values, respectively.

†Compared with never smokers. 
TABLE E5. Adjusted odds ratios (95\% Cls) of current asthma, wheeze, asthma attack, and asthma-related ED visit with increased FENO and B-Eos values after adjusting for age, sex, BMI, smoking history, and recent use of ICSs or OCSs

\begin{tabular}{llcccc}
\hline FENo value & B-Eos value & Asthma & Wheeze & Asthma attack & Asthma-related ED visit \\
\hline Normal & Normal & 1 & 1 & 1 & 1 \\
Normal & Intermediate & $1.35(1.09-1.67)$ & $1.44(1.23-1.68)$ & $1.61(1.21-2.15)$ & $1.52(0.94-2.48)$ \\
Normal & High & $1.87(1.34-2.61)$ & $1.48(1.13-1.94)$ & $2.13(1.38-3.28)$ & $2.17(1.09-4.32)^{*}$ \\
Intermediate & Normal & $1.65(1.26-2.16)$ & $1.35(1.08-1.69)$ & $1.50(1.02-2.22)^{*}$ & $0.76(0.32-1.81)$ \\
Intermediate & Intermediate & $2.24(1.60-3.12)$ & $1.90(1.44-2.50)$ & $2.62(1.70-4.04)$ & $2.37(1.15-4.87)^{*}$ \\
Intermediate & High & $2.59(1.57-4.26)$ & $2.68(1.76-4.08)$ & $3.51(1.94-6.34)$ & $3.52(1.45-8.50)$ \\
High & Normal & $2.76(1.66-4.59)$ & $1.96(1.25-3.08)$ & $1.97(0.90-4.31)$ & $0.74(0.10-5.51)$ \\
High & Intermediate & $2.61(1.71-3.99)$ & $3.00(2.10-4.28)$ & $4.08(2.52-6.59)$ & $1.90(0.77-4.69)$ \\
High & High & $5.86(4.07-8.44)$ & $4.99(3.56-7.00)$ & $6.22(3.99-9.68)$ & $2.96(1.39-6.30)$ \\
\hline
\end{tabular}

Multiple logistic regression analyses were performed, and adjusted ORs (95\% CIs) are presented. All strata are compared with the stratum with normal FeNo and B-Eos values. A relation is significant if the CI does not include 1 .

* Significance not consistent after adjusting for multiple comparisons (Holm method). 